\title{
A closer look at four dot masking of a foveated target
}

Marwan Daar, Hugh R Wilson

Four dot masking with a common onset mask was recently demonstrated in a fully attended and foveated target (Filmer et al., 2015). Here, we replicate and extend this finding, by directly comparing a four dot mask with an annulus mask while probing masking as a function of mask duration, and target-mask separation. Our results suggest that while an annulus mask operates via spatially local contour interactions, a four dot mask operates through spatially global mechanisms. We also measure how the visual system's representation of a oriented bar is impacted by a four dot mask, and find that masking here does not degrade the precision of perceived targets, but instead appears to be driven exclusively by rendering the target completely invisible. 


\title{
A closer look at four dot masking of a foveated target.
}

\author{
Marwan Daar ${ }^{1} \&$ Hugh Wilson ${ }^{1}$
}

Four dot masking with a common onset mask was recently demonstrated in a fully attended and foveated target (Filmer et al., 2015). Here, we replicate and extend this finding, by directly comparing a four dot mask with an annulus mask while probing masking as a function of target-mask separation. Our results suggest that while an annulus mask operates via spatially local contour interactions, a four dot mask operates through spatially global mechanisms. We also measure how the visual system's representation of an oriented bar is impacted by a four dot mask, and find that masking here does not degrade the precision of perceived targets, but instead appears to be driven exclusively by rendering the target completely invisible.

1: Centre for Vision Research, York University, Canada. 


\section{Introduction}

Over the last two decades, common onset masking with a four dot mask (also referred to as object substitution masking) has proven to be a valuable way to study the processes by which a visual object is consciously rendered (Goodhew, Pratt, Dux, \& Ferber, 2013). In this masking paradigm, a target is briefly flashed along with four surrounding dots, the latter of which persist for a variable duration. As this mask duration increases, target visibility is reduced, a phenomenon that likely reflects competition between the target and mask. Given the sparse nature of this mask, relative to more traditional masks that either fully surround the target's contours or spatially camouflage it, four dot masking is thought to be a powerful demonstration of interactions at a spatially global object level, rather than of spatially local contour interactions (Di Lollo, Enns, \& Rensink, 2000). In addition to providing insights into the time-course of visual processing, four dot masking offers a means to probe the way in which objects are individuated by the visual system (Goodhew, Edwards, Boal, \& Bell, 2015; Lleras \& Moore, 2003). It also allows us to examine how attentional manipulations can bias competition between visual objects, such as a target and mask (Pilling, Gellatly, Argyropoulos, \& Skarratt, 2014; Tata \& Giaschi, 2004), and to explore the ability of a masked target to influence subsequent processing (Choo \& Franconeri, 2010; Goodhew, Visser, Lipp, \& Dux, 2011).

Until recently, four dot masking was only reliably reported when the target was presented in peripheral visual field, usually as part of a set of possible targets. Masking has also been found with a single target in peripheral visual field, but only reported in cases where the spatial location of this target was randomized (Argyropoulos, Gellatly, Pilling, \& Carter, 2013; Camp, Pilling, Argyropoulos, \& Gellatly, 2015). A recent report, however, demonstrates four dot masking involving a fully attended target in central visual field (Filmer, Mattingley, \& Dux, 2015). This important finding clearly shows that neither distributed attention nor crowding are necessary conditions for this form of masking. The ability to study four dot masking in central visual field with a single target is valuable for a few reasons. With multiple targets, there is the risk of noise introduced by pooling data across multiple target locations. With a single target location, one can therefore obtain well controlled data with relatively fewer trials. Furthermore, the use of a single, central target allows for efficient use of space within the visual field, and there are thus fewer limitations on parameters such as target size, and targetmask separation. Finally, the use of a single target offers the theoretical convenience of removing the influence of crowding, and feature misbinding (mistaking a feature of one of the distractors for a feature of the target), on any discovered effects.

In the current study, we sought to explore four dot masking of a foveated target more closely. We were interested in two questions. First, how does a four dot mask compare with an annulus mask, as a function of target-mask separation? As an annulus completely surrounds the target, it can mask the target through local inhibition as well as through object substitution/updating (Enns, 2004). It is unlikely, however, that a four dot mask operates via local inhibition, given its sparse nature. Comparing these two mask types may provide evidence for a dissociation of these mechanisms. Second, we were interested in how this form of masking affects the target representation (Agaoglu, Agaoglu, Breitmeyer, \& Öğmen, 2015; Harrison et al., 2016). To do this, we developed a matching task where observers adjusted the orientation of a bar to match that of the target bar, and examined how the distribution of 
43 errors changed between baseline and masking conditions. This task also allowed us to examine whether

44 the errors were more likely to occur when the target bar was closest to any of the four dots ${ }^{1}$. If so, then

45 this would point to the contribution of local masking mechanisms.

\section{Experiment 1}

47 Our first step was to see whether we could successfully replicate the masking effect found in Filmer et 48 al. (2015). In their study, they used a forward noise mask in addition to a common onset trailing four

49 dot mask, and found a reliable performance drop as the duration of the four dot mask increased.

\section{Methods \& Procedures}

The target was an annulus with a bar projecting from its centre to one of four cardinal points along the circumference. The inner radius of this annulus was $32.3 \mathrm{arcmin}$, and the outer radius was 39.5 arcmin. The projected bar, whose length was equal to the inner radius of the annulus, had a thickness of 5 arcmin. The mask comprised four small circles (diameter $=14.4$ arcmin) located at the corners of an imaginary square concentric with the target. The separation of the mask, measured from the edge of each dot to the outer edge of the target annulus, was 31.6 arcmin. The forward mask was a circular patch of Gaussian noise, with a mean luminance equal to the that of the background, and whose radius was equal to the outer radius of the target annulus. A square cross of length 28.7 arcmin was used for fixation. All stimuli were centrally presented with no spatial jitter. The target and four dot mask are depicted in Figure 1.

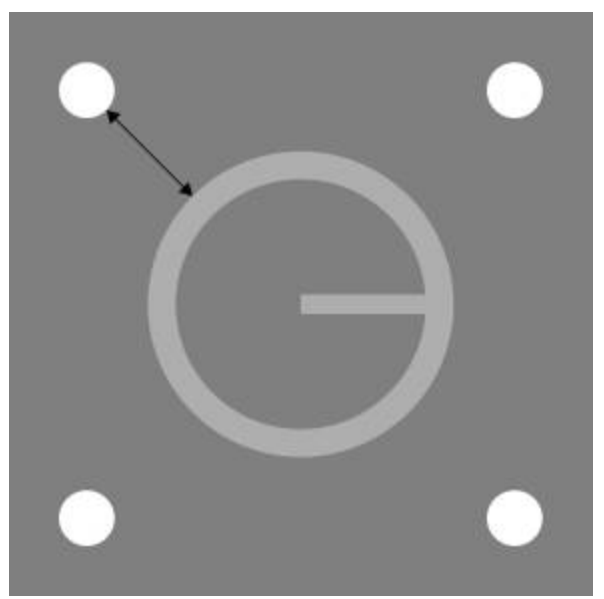

Figure 1. Target and four dot mask drawn to scale. The black line indicates the distance along which target-mask separation was measured. The separation shown here was used in Experiments 1 and 2 .

1 We would like to thank Haluk Öğmen for providing the suggestion for this experiment. 
61 The luminance of the target, as measured with a Konica-Minolta LS-100 (integration time: $400 \mathrm{~ms}$ ) 62 was $65.8 \mathrm{~cd} / \mathrm{m}^{\wedge} 2$, while that of the four dot mask and fixation cross was $97 \mathrm{~cd} / \mathrm{m}^{\wedge} 2$. All stimuli were 63 presented against a uniform background grey field of luminance $47 \mathrm{~cd} / \mathrm{m}^{\wedge} 2$, on a VIEWPixx display calibrated to linear light (gamma $=1)$, at a viewing distance of $1.28 \mathrm{~m}$ in a dimly lit room. The display was running at $120 \mathrm{hz}$ in scanning backlight mode. In this mode, the backlight is scanned down the display in synchrony with the updated pixels, and the pixel rise time (black to white) and fall time (white to black) are both $1 \mathrm{~ms}$. Thus, in a single frame comprising a white field, each pixel lets light through (above and beyond the black level luminance) for only $2 \mathrm{~ms}$. It is important to precisely specify the mode in which visual content is rendered on the display, as this has implications for the actual stimulus durations, which are not always readily calculable based on reported frame rate (Elze, 2010).

The trial sequence is shown in Figure 2A. Each run was initiated with a keypress, at which point the fixation cross appeared for one second. As soon as the fixation disappeared, the forward mask appeared, lasting $200 \mathrm{~ms}$. Immediately following its offset were the target and four dot mask (henceforth termed "4DM"). The target persisted for $8.3 \mathrm{~ms}$. In the common offset condition, which served as a baseline, the mask disappeared with the target (mask duration $=8.3 \mathrm{~ms}$ ). Two other mask durations were tested: $\mathbf{2 5 0} \mathbf{~ m s}$, and $\mathbf{5 0 0} \mathbf{~ m s}$. Upon mask offset, the background remained visible, and a keypress response indicated which of the four directions the observer believed the line to be pointing along (up, down, left, or right). The task was self paced, and keypress responses initiated the next trial. Within each run, each of the three mask durations was repeated 30 times in random order, and observers completed three runs, for a total of 90 trials per condition. Seven observers completed this experiment, one of whom is the first author, and the rest of whom were naive. All observers, in this and subsequent experiments, gave verbal consent before participation. This study was approved by the Human Participants Review Committee (HPRC) at York University (approval number HPRC 2014094). Before beginning these experimental runs, each observer completed a PEST procedure (Taylor \& Creelman, 1967) for the common offset condition, where the standard deviation of the noise patch was varied until 80 percent performance was achieved. This value was then used in the main experiment for that observer across all conditions. Feedback was not provided in either the PEST phase or the experimental trials. 
A

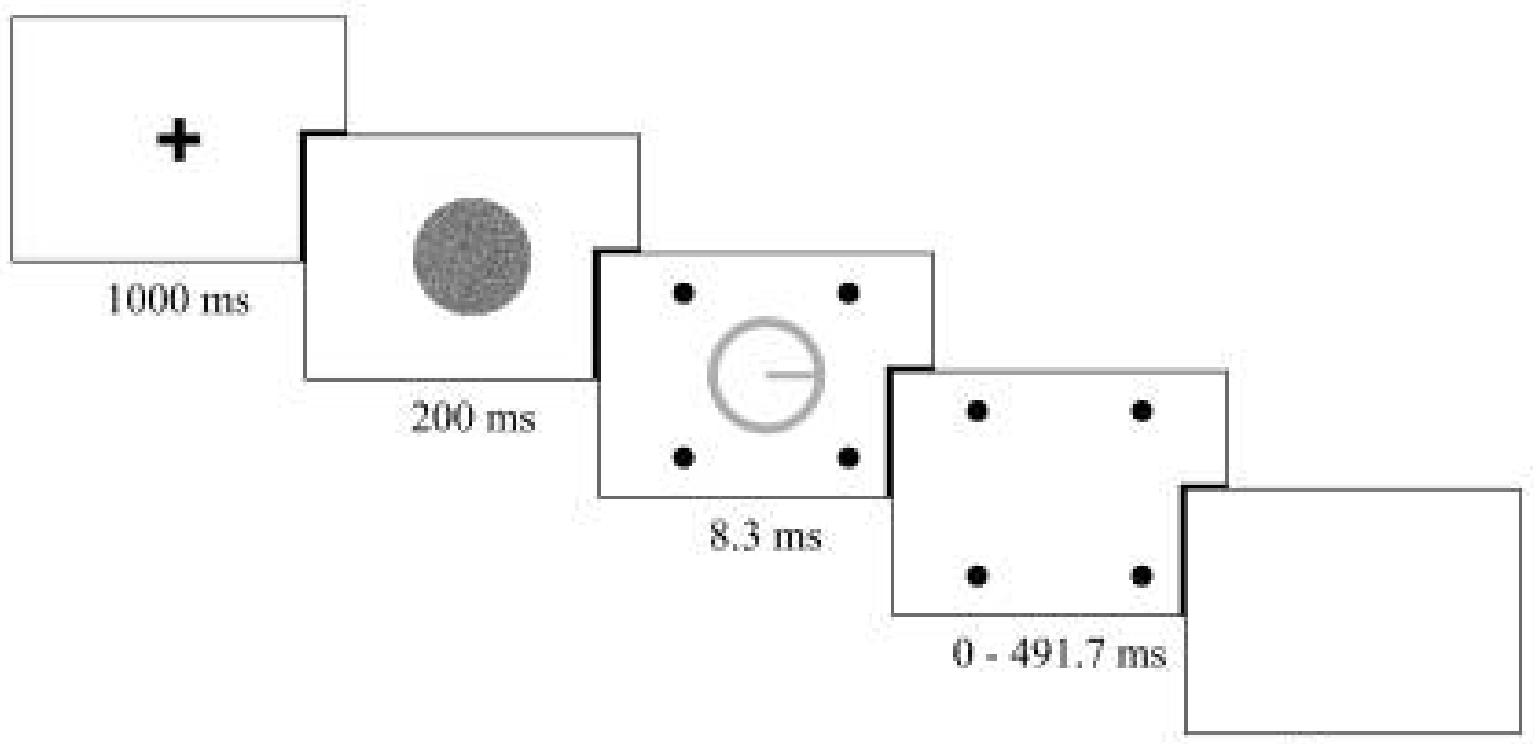

until response

B

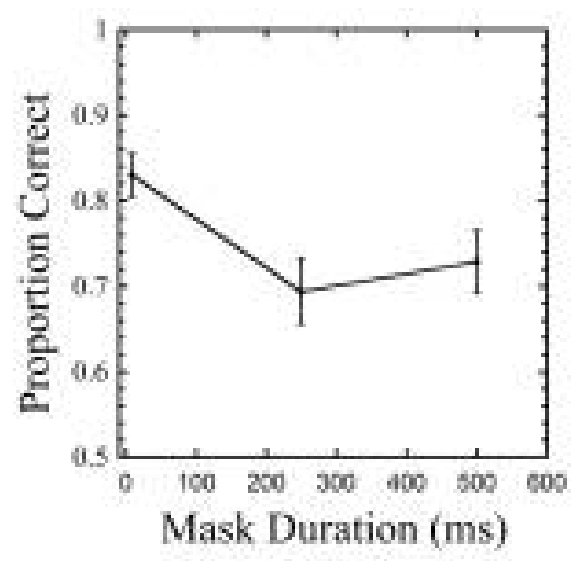

Figure 2. Trial sequence and results for Experiment 1. Stimuli here and in all subsequent figures are rendered in reverse contrast. Error bars indicate standard errors.

\section{Results \& Discussion}

91 Results are shown in Figure 2B. A one way repeated measures ANOVA revealed a main effect of mask

92 duration $(\mathrm{F}(2,12)=4.613, \mathrm{p}=0.033)$. Follow up comparisons showed a difference between the

93 common offset and 250 ms mask duration conditions $(t(6)=3.985, p=0.007)$, no difference between

94 common offset and $500 \mathrm{~ms}(\mathrm{t}(6)=1.942, \mathrm{p}=0.1)$, and no difference between $250 \mathrm{~ms}$ and $500 \mathrm{~ms}(\mathrm{t}(6)$

$95=0.681, \mathrm{p}=0.521)$. 
96

97

98

99

100

101
103

104

105

106

107

108

109

110

111

112

113

114

115

116

117

118

119

120

Experiment 1 shows a clear masking effect, with about a 13.5 percent drop in performance between the common offset and $250 \mathrm{~ms}$ mask duration conditions. The data do not provide strong evidence for masking at the $500 \mathrm{~ms}$ condition, nor do they support recovery from masking (Goodhew, Dux, Lipp, \& Visser, 2012), as there was no difference between the $250 \mathrm{~ms}$ and $500 \mathrm{~ms}$ conditions. In our next experiment, we measured masking as a function of target-mask separation, with both a 4DM and an annulus mask.

\section{Experiment 2}

Our next experiment compared a 4DM with an annulus mask as a function of the separation between target and mask. If the annulus mask is acting primarily through local inhibitory mechanisms, while the 4DM involves object-level mechanisms, then as target-mask separation increases, one would expect a drop in annulus masking, while dot masking should remain relatively unchanged.

\section{Methods \& Procedures}

The general procedure was similar to the previous experiment. Here, runs were blocked according to mask type (4DM, annulus, see Figure 3). Within each run, four different mask separations were tested, with a constant mask duration of $250 \mathrm{~ms}$ (7.2 $\mathbf{a r c m i n}, 17.2 \mathrm{arcmin}, \mathbf{2 7 . 3} \mathrm{arcmin}$, and 103.5 arcmin), in addition to a common offset condition for each mask at the lowest separation (7.2 arcmin). Each of these five conditions was tested in random order, 30 times per run, and observers completed three runs for each mask type, for a total of 90 trials per mask type per separation. Nine observers completed this experiment, all of whom were naive. Observers completed runs in alternating order of mask type. Four of them began with the annulus mask, and five began with the 4DM. Instead of running a PEST procedure, observers were trained in the common offset condition, with increasing amounts of noise in the forward mask. This was done until the experimenter had established a noise level at which performance was around 75 percent correct. In these training trials, a correct response generated an auditory tone. In the experimental trials, in this and in all other experiments in this study, no feedback was provided. 


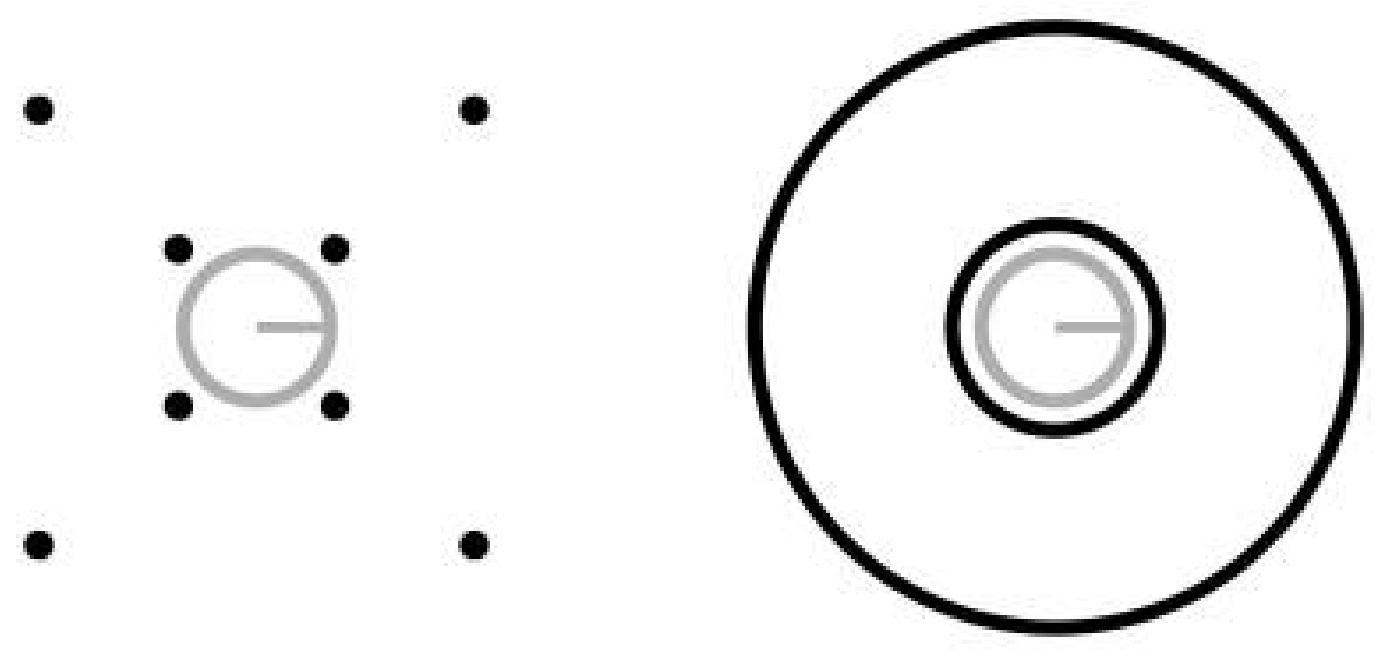

Figure 3. Stimuli used in Experiment 2. Left: Four dot mask. Right: Annulus mask. Each mask type is shown with both the smallest (7.2 arcmin) and largest (103.5 arcmin) target-mask separations. Stimuli are drawn to scale.

\section{Results \& Discussion}

Results are shown in Figure 4. A two way repeated measures ANOVA (first factor = mask type: two levels (4DM, annulus); second factor = target-mask separation: four levels, excluding baseline) revealed no main effect of mask type $(\mathrm{F}(1,8)=1.218, \mathrm{p}=0.3)$, a main effect of separation $(\mathrm{F}(3,24)=$ $7.489, p=0.001$, and an interaction between mask type and separation $(F(3,24)=3.245, p=0.04)$. To verify that masking was obtained with the 4DM, a paired samples t-test was used to compare the 7.2 arcmin 250 ms mask with the baseline condition (7.2 arcmin common offset mask), and this confirmed masking $(\mathrm{t}(8)=2.861, \mathrm{p}=0.021)$. The striking pattern found with the annulus mask is strong evidence of the primacy of spatially local inhibitory mechanisms here. By a separation of 27.3 arcmin, annulus masking was greatly attenuated, compared to the 7.2 arcmin mask $(\mathrm{t}(8)=3.067, \mathrm{p}=0.015)$. In contrast, the 4DM showed no change in masking between the 7.2 and $27.3 \operatorname{arcmin}$ conditions $(\mathrm{t}(8)=0.77, \mathrm{p}=$ 132 0.47). 
135 increasing the separation of the individual dots, as was done in our current experiment (Di Lollo, Enns, 136 \& Rensink, 2000). In that study, masking did not decrease, up to the measured separation of 40 arcmin, 137 a finding that closely matches our own. These findings support the idea that (spatially sensitive) local 138 inhibitory mechanisms underlie annulus masking, while (less spatially sensitive) object level 139 mechanisms underlie dot masking.

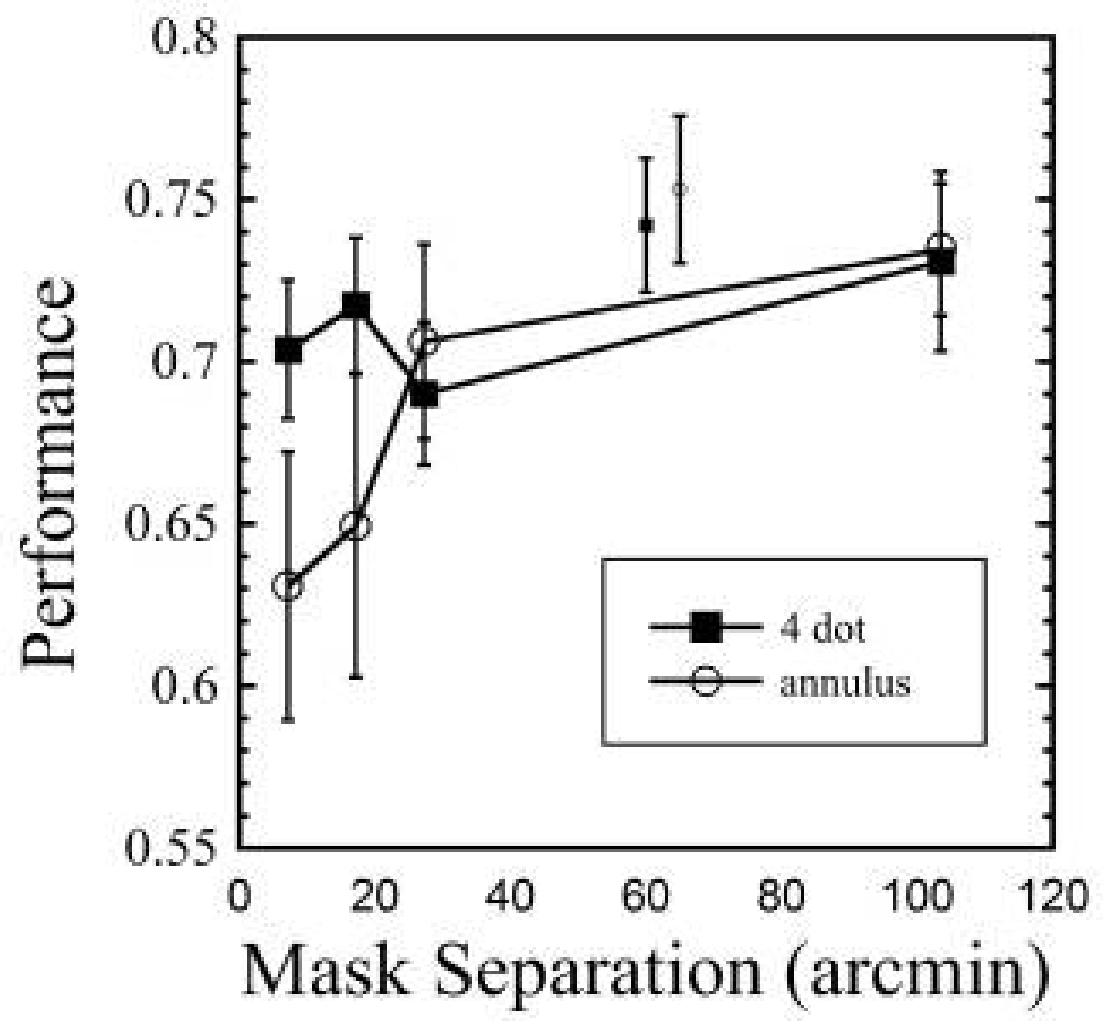

Figure 4. Results from Experiment 3, showing performance across nine observers for both mask types as a function of separation from target. The two smaller symbols in the upper middle region indicate baseline values for the two masks (common offset mask, 7.2 arcmin separation). Error bars indicate standard errors.

\section{Experiment 3}

The two previous experiments had observers select from four possible line orientations. In our final experiment, which only used a 4DM, the task was to adjust the orientation of a reference line until it matched one of 90 possible target orientations. This allowed us to investigate two separate questions. First, are errors more likely to occur when the target line is oriented such that it is closer to one of the four dots? If so, then this would point towards the contribution of spatially local masking mechanisms. Second, how does the distribution of errors change between baseline and masked conditions? Is the 
147

148

149

150

151

152

representation of a masked target simply less precise (in the orientation domain), or is it never consciously processed, in which case observers would have to guess? Or is it some combination of both? By modelling the errors as a mixture of a Gaussian distribution centred on the actual target orientation (representing trials in which the target was perceived) and a Uniform distribution across all possible orientations (representing trials in which observers were relegated to guessing), we were able to address this question.

\section{Methods \& Procedures}

The stimuli were similar to the previous experiments; however, the target could now adopt any of 90 unique orientations, spaced at $4^{\circ}$ intervals around the circle, ranging from $0^{\circ}$ to $356^{\circ}$. Observers used the mouse to adjust and select the orientation of a reference pattern, which appeared $500 \mathrm{~ms}$ after target offset. The reference pattern and the target were visually identical, except that the orientation of the target was determined by the current trial, whereas the orientation of the reference pattern was determined by the current mouse position.

Each run contained three conditions. Target alone with no mask, a common offset 4DM, and a $250 \mathrm{~ms}$ duration 4DM. The target-mask separation was 10.8 arcmin. Within each run, each condition was tested once at each of the 90 orientations, for a total of 270 trials per run, which were presented in random order. Within each run, a message appeared every 90 trials, indicating the current progress, at which point a mouse click returned the observer to the trial flow. Each observer completed three runs, thus each orientation was tested three times per condition. Nine observers completed this experiment, one of whom is the first author and the rest of whom were naive. Training was done in the common offset condition, and for each observer, the experimenter increased the standard deviation of the noise patch until performance was about 60 percent correct. In this training phase, a correct response was defined as being within 20 degrees of the actual target orientation, and was signalled with a tone.

\section{Results \& Discussion}

The purpose of this experiment was two-fold. First, we first investigate whether there is a bias for increased masking when the orientation of the target line was closer to the four dots. Second, we model the distribution of errors for each of the three masking conditions.

For each trial, the difference between the actual target orientation and the reported orientation was calculated. Trials were pooled into two categories: those in which the presented target line orientation was aligned in proximity to the dots (oblique), and those in which it was aligned along the cardinal axes (cardinal), and thus maximally distant from the dots. Classification was done by visual inspection of a modified target pattern, which was identical to the experimental pattern, except that the oriented bar now extended beyond the annulus (Figure 5A). Upon inspection of each of the 90 bar orientations, if any part of the bar intersected any of the four dots, the orientation was classified as oblique. Cardinal orientations were classified with a similar procedure, but with the four dots arranged in a diamond pattern (see Figure 5A for a depiction of one of these dots). Of the set of 90 orientations, 18 orientations were classified as oblique, and 18 as cardinal. This meant that for each observer, there were 54 oblique trials and 54 cardinal trials for each of the three masking conditions. Results are shown in Figure 5B. Note that the errors are unsigned. The overall errors indicate the mean of all 90 orientations. 

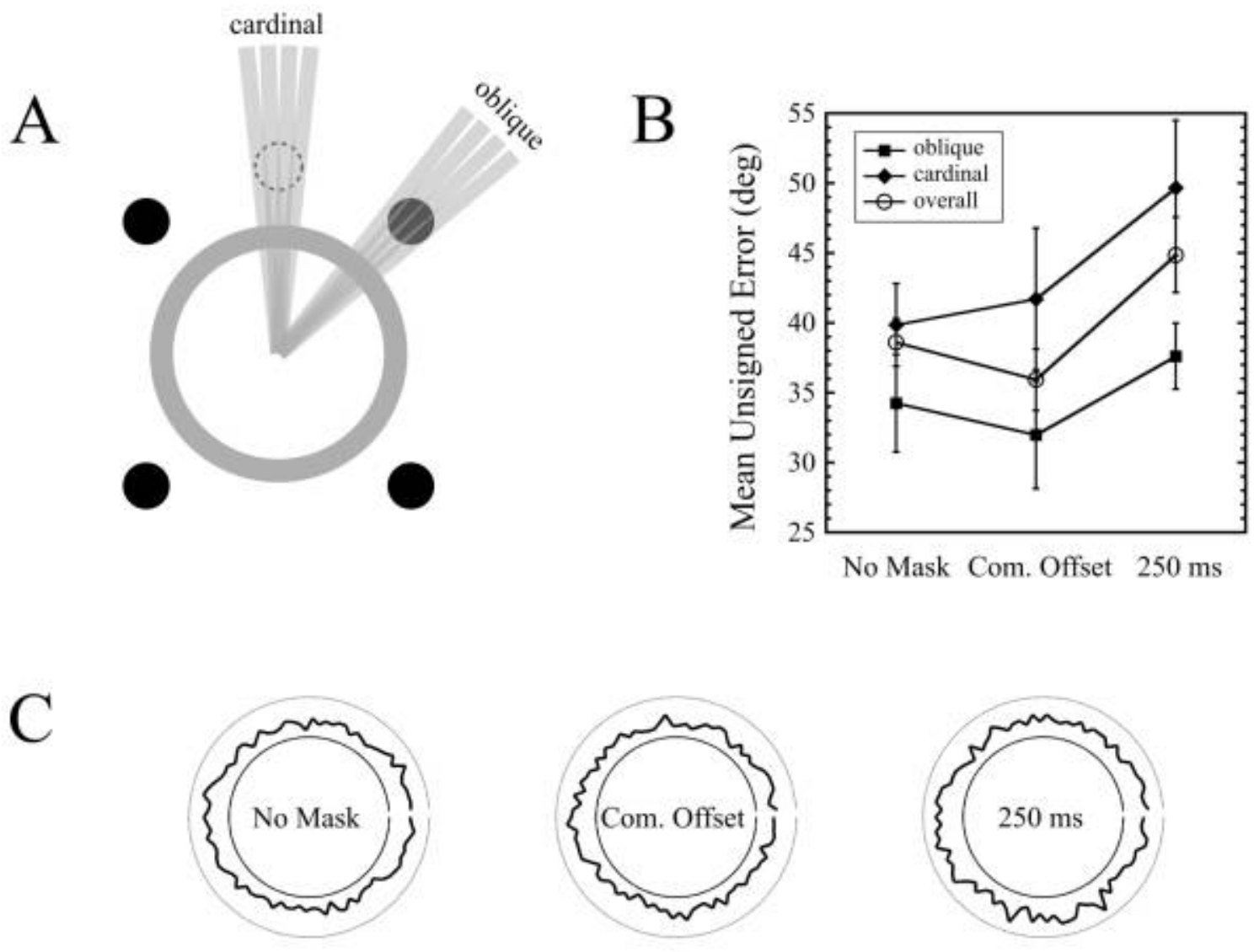

Figure 5. Orientation bias results from Experiment 4. A: Depiction of how oblique and cardinal orientations were defined. The dot shown with the dashed outline served as a marker to classify orientations as oblique (only one of these dots is shown in the figure). B: Errors as a function of mask condition and orientation type (oblique vs. cardinal) across nine observers. Error bars indicate standard errors. C: Polar plots depicting errors as a function of target orientation, for each mask type, across all observers. The magnitude of the error is depicted by the radially projected distance from the inner circle to the thick black curve. The outer circle indicates the error expected by chance performance (90 degrees).

The first thing to note is that masking did occur. The overall error for the common offset condition was 35.9 degrees, while that of the $250 \mathrm{~ms}$ mask was 44.9 degrees, and this difference was significant $(\mathrm{t}(8)=4.122, \mathrm{p}=0.003)$. Interestingly, the presence of a common offset mask increased performance relative to no mask at all $(\mathrm{t}(8)=3.822, \mathrm{p}=0.005)$.

If the (trailing) 4DM was exerting its effect through local spatial mechanisms, then a greater increase of errors in the oblique orientations in the $250 \mathrm{~ms}$ condition (relative to the common offset baseline) would be expected, compared to the increase in cardinal errors. To test this interaction, we ran a two way repeated measures ANOVA (first factor = mask condition: two levels (common offset, 250 
195

196

197

198

199

200

201

202

203

204

205

206

207

208

209

210

211

212

213

214

215 EQ 1:

$$
P D F=W_{G} * G(\mu, \sigma)+\left(1-W_{G}\right) * U(-\pi, \pi)
$$

216

217

218

219

220

221

222

223

224

225 perceived target trials, respectively. is defined below in Equation 1: $\mathrm{ms}$ ); second factor $=$ target orientation: two levels (oblique, cardinal)). This revealed a strong trend for the effect of mask condition $(\mathrm{F}(1,8)=4.825, \mathrm{p}=0.059$; no main effect of error location $(\mathrm{F}(1,8)=$ $3.549, \mathrm{p}=0.096$; and no interaction $(\mathrm{F}(1,8)=0.138, \mathrm{p}=0.72)$.

Our data do not show evidence of an orientation bias in masking: we did not observe increased masking at target orientations proximal to the dot locations, as evidenced by the almost perfectly parallel lines in the right half of Figure 5B. While this does not definitively prove that dot masking operates via spatially global mechanisms, it is consistent with the finding in Experiment 2 that dot masking is, to a large extent, independent of target-mask separation.

For any trial in which masking occurred, there are at least two possible scenarios. On the one hand, the target may have never been consciously processed, either because the representation was completely obliterated, or because it had been degraded below a critical threshold. In such a scenario, an observer would have to guess the orientation of the target. On the other hand, the target representation may have remained consciously accessible, but in a degraded form. If the nature of this impoverishment was such that the precision of orientation information was compromised, then we would expect a reduction in the precision of the response. Note that these possibilities are not mutually exclusive. We fit a mixture model to our data to estimate the relative proportions of trials that corresponded to these two possibilities, using a Gaussian and Uniform for the perceived target and non

The probability density function for this mixture model, which defines the distribution of signed errors,

where $\mathrm{G}$ is a Gaussian function, with mean $(\mu)$ and standard deviation $(\sigma)$, and $\mathrm{U}$ is a Uniform distribution along the specified interval. $\mathrm{W}_{\mathrm{G}}$ is the weight of the Gaussian term, and as the mixed distribution sums to unity, the weight of the Uniform term is $1-\mathrm{W}_{\mathrm{G}}$. In the context of our experiment, a lower value of $\mathrm{W}_{\mathrm{G}}$ indicates more guessing, and a higher value of $\sigma$ indicates less precise responses of perceived targets. This mixture model is very similar to that used by Zhang \& Luck (2008), except they used a circular normal distribution, whereas we used a normal distribution. While a circular normal distribution would be a better match for the circular nature of our error variable, which spanned $-180^{\circ}$ to $180^{\circ}$, it has been shown that a single Gaussian is an excellent approximation to a circular normal distribution when the standard deviation is small relative to the range of possible errors (Shooner, Tripathy, Bedell, \& Öğmen, 2010; also see Appendix A in Agaoglu et al., 2015).

For each observer, and for each of the three masking conditions (no mask, common offset, 250 ms mask), a maximum likelihood procedure (Myung, 2003) was used to estimate $\mathrm{W}_{\mathrm{G}}, \mu$, and $\sigma$. The results, pooled across all nine observers, are shown in Figure 6. A one way repeated measures ANOVA was run on each of the two parameters, $\sigma$ and $\mathrm{W}_{\mathrm{G}}$, across all three masking conditions. For $\sigma$, there was no main effect of masking condition $(F(2,16)=0.046, p=0.956)$. For $\mathrm{W}_{\mathrm{G}}$, a main effect of masking condition was found $(\mathrm{F}(2,16)=12.215, \mathrm{p}=0.001)$. Interestingly, while the guess rate with the 
232 trailing mask was greater than in the common offset condition $(\mathrm{t}(8)=4.664, \mathrm{p}=0.002)$, it was also

233 higher in the no mask condition than in the common offset condition $(t(8)=3.985, p=0.004)$.

A

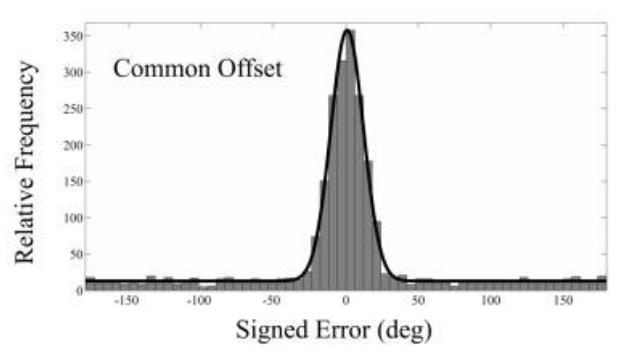

$\mathrm{B}$

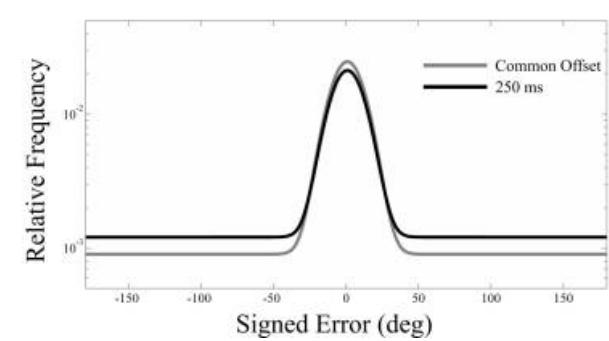

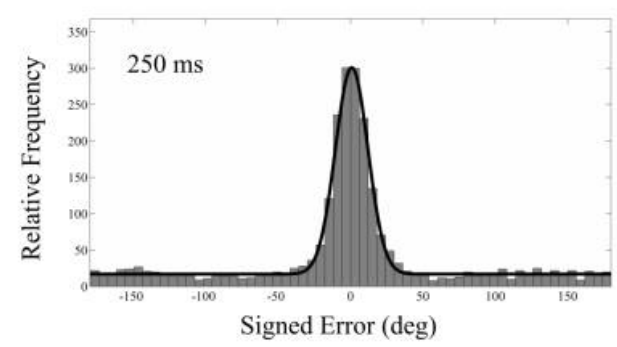

$\mathrm{C}$

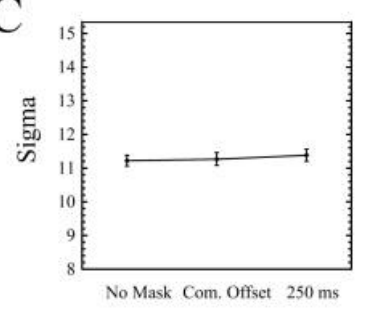

$\mathrm{D}$

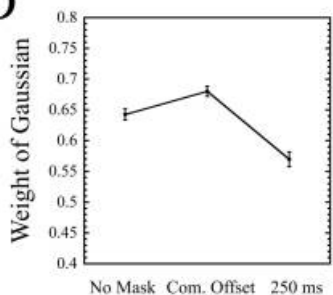

Figure 6. A: Distribution of errors across nine observers for the common offset and $250 \mathrm{~ms}$ conditions, along with model fits. B: Model fits for the error distributions of the common offset mask and $250 \mathrm{~ms}$ mask (note the log scale). Each curve represents a mixture of a Gaussian and Uniform. Note that the width of the Gaussian remains unchanged between these two masking conditions. C: Standard deviation of Gaussian component for each of the three mask conditions. D: Weight of Gaussian component for each of the conditions. Error bars indicate standard errors.

234

Masking appears to have absolutely no effect upon the precision of responses (Figure 6C). Instead, the performance drop between the common offset mask and the trailing mask (Figure 5B) is driven entirely by the target being rendered invisible, where the guessing rate increased from about $32 \%$ to $43 \%$ (Figure 6D). This also demonstrates that the $250 \mathrm{~ms}$ mask had a measurable impact, relative to the common offset mask, on only a fraction of the trials.

These results are fairly striking, especially when compared against two recent studies that conducted very similar experiments (Agaoglu et al., 2015; Harrison et al., 2016). In particular, Agaoglu et al. used an oriented bar as a target stimulus very similar to ours, and, using a backward masking paradigm, found that the standard deviation of observers' responses increased from about 11 degrees to as high as 20 degrees, depending upon the type of mask being used (all mask types also increased the guessing rate). On the other hand, the standard deviation of the responses in our experiment remained at around 11 degrees across all masking conditions. Harrison et al. (2016), using a similar masking paradigm, where observers attempted to match the orientation of a gap in a target annulus, found that the standard deviation of responses showed about a 40 percent increase in the trailing vs. common offset condition. We will explore this discrepancy further in the general discussion. While we found no 
evidence for an effect of masking condition upon response precision, our analysis does not directly say anything about the evidence against an effect. To address this, we ran a Bayesian analysis on our data, and have presented this in the Appendix.

\section{General Discussion}

In Experiment 1, we successfully reproduced four dot masking in a fully attended and foveated target, as originally reported by Filmer et al. (2015). The data from Experiment 2, which directly compared a 4DM with an annulus mask, strongly suggest that these two mask types operate through at least partially distinct mechanisms. Experiment 3 demonstrates that in the context of our stimuli and task, there is no evidence that the encoding precision of the target (in the orientation domain) is affected by masking. Rather, masking appears to be driven exclusively by rendering the target inaccessible to conscious processing. In this section, we will explore these findings in more depth.

As in Filmer et al. (2015), we included a forward noise mask in our study. It is an open question, however, whether similar results would obtain had we incorporated the noise mask into the same frame as the target. In the current experiments, while the stimulus onset asynchrony (SOA) of the noise mask, relative to the target, was $-200 \mathrm{~ms}$, the onset of the target occurred immediately following the offset of the noise mask (i.e. if we take into account the scanning mode of our display, this would correspond to an interstimulus interval (ISI) of slightly below $8.3 \mathrm{~ms}$ ). As such, the noise mask and the target would likely be integrated into a unified percept. Inspection of neurophysiological data in Macknik \& Livingstone (1998) suggests that the transient response to the target is slightly lower with an SOA of $-100 \mathrm{~ms}$ (ISI of $0 \mathrm{~ms}$ ), relative to a common onset common offset condition (Figure 4 in their paper). However, the masks in that study comprised a set of flanking bars. A more relevant study (Agaoglu et al., 2015), which used an almost identical task to ours, showed peak masking with a noise mask with an SOA of $0 \mathrm{~ms}$, which appeared to show even greater masking than that found with a forward mask of SOA -10 ms (ISI = $0 \mathrm{~ms}$ ). Thus, although we have not tested this, we suspect that the critical element that enables successful four dot masking here is the incorporation of noise into the target, rather than the use of a forward mask.

Why would a noise mask be critical for four dot masking? Its ostensible purpose in Filmer et al. (2015) was to reduce baseline performance to below ceiling. Pilot work in our lab, however, showed no evidence of four dot masking (in the absence of a noise patch) when the contrast of the target was reduced such that baseline performance was well below ceiling. Indeed, if ceiling effects were the only issue here, then it is surprising that it has taken almost two decades to produce reliable four dot masking in a fully attended and foveated target (although see Dux, Visser, Goodhew, \& Lipp, 2010). Rather, it is more likely that the target needs to be degraded in a specific manner. With the use of a noise mask, the integrity of the stimulus as a coherent object is dramatically compromised. In order to perceive an oriented bar, perceptual filling in processes may be required, and this may delay or otherwise compromise the formation of a stable object representation, which in turn may leave the target more vulnerable to being dominated by the mask. Another possibility is that if the 4DM is able to disrupt the perception of target elements proximal to the dots, as is the case, for example, in object trimming (Kahan \& Enns, 2010), this may sometimes leave less information available for the visual system to glean the bar's orientation, if the part of the object being trimmed happens to contain useful information. This would be equivalent to reducing the signal to noise ratio. Both of these possibilities are compatible with the finding in Experiment 3 that suggests when an object is successfully masked 
291 here, it is rendered invisible.

292

293

294

295

296

297

298

299

300

301

302

303

304

305

306

307

308

309

310

311

312

313

314

315

316

317

318

319

320

321

322

323

324

325

326

327

328

329

330

331

332

333

It should be noted that while Experiment 1 was very similar to that in Filmer et al. (2015), it was not an exact replication, as there were differences in the dimensions and contrast of the stimuli. In particular, while the target and mask in Filmer et al.'s first experiment had the same contrast relative to the background, we used a lower contrast for the target, compared to the mask. Our stimuli are thus more comparable to Filmer et al's second experiment, where the contrast of the target was thresholded to the desired performance level.

The data from our second experiment provides evidence for a number of related ideas. First, the finding that dot masking did not attenuate with increasing target-mask separation suggests that four dot masking operates via mechanisms that are relatively insensitive to spatially local interactions between target and mask. Rather, masking here likely involves mechanisms that primarily involve representations at higher levels of the visual processing hierarchy, where receptive fields are larger (Dumoulin \& Wandell, 2008; Smith, Singh, Williams, \& Greenlee, 2001). While the involvement of higher visual areas is compatible with object level accounts involving interference between feedback and ongoing input, our data do not directly say anything about whether feedback is involved. Second, the finding that annulus masking did attenuate with increasing target-mask separation is strong evidence that here, the mechanisms are sensitive to spatially local interactions between the target and the mask, and confirms the findings of many previous studies that have found a similar sensitivity to spatial separation in the literature on metacontrast masking (see § 2.6.6 in Breitmeyer \& Öğmen, 2006). Finally, the interaction between mask type and separation adds to the evidence that four dot masking operates through mechanisms that are at least partially unique from those involved with masks whose contours fully surround the target.

In our final experiment, we found that masking appears to be driven exclusively by rendering the target completely invisible. While this finding is certainly compatible with the idea of object updating (Pilling \& Gellatly, 2010), where the original object (target + mask) is updated into a new object (mask), it is not proof of object updating. Another possibility is that four dot masking simply degrades the target without impacting the encoding precision of its orientation information. For example, if the mask is simply reducing the contrast of the target, then it is possible that orientation encoding is unaffected. Mareschal \& Shapley (2004) found that orientation discrimination of foveally presented gratings was unaffected by contrast when the diameter of these gratings was large (1 degree of visual angle). However, for two out of the three observers in their experiment, contrast did have a dramatic impact on these thresholds when the diameter was as large as 0.5 degrees (all of the observers showed reduced performance as contrast was reduced when the diameter was 0.25 degrees). The length of the oriented bar in our experiment was about 0.5 degrees, and it is certainly possible that our cohort of observers was demonstrating contrast invariance to orientation encoding with this stimulus size. It would be interesting to run our experiment with a bar length of 0.25 degrees and see if precision is similarly unaffected. Indeed, in Filmer et al. (2015), the diameter of the target was 0.55 degrees, which meant that the bar length was about 0.25 degrees. Another alternative to object updating is object trimming (Kahan \& Enns, 2010), where a two dot mask was shown to interfere with target contours that were adjacent to these dots. If object trimming was occurring in our study, then, as discussed earlier, this could account for the target being rendered invisible due to a reduced signal to noise ratio. However, if this were the case, then we might expect more masking when the target orientation was aligned with the oblique axes, and we found no evidence of this (Figure 5B). 
Another aspect of Experiment 3 worth considering is that, while in our study, we found no change in response precision, two other similar studies showed a substantial drop in response precision (Agaoglu et al., 2015; Harrison et al., 2016). However, there are some important differences to consider here. In Harrison et al., the target was an annulus whose gap could adopt a number of different orientations. It is possible that the orientation specific information in such a stimulus may be compromised, while that of an oriented bar would not, given the same general type of mask induced degradation. For example, it is clear how object trimming could result in part of the circle being occluded near the gap, resulting in a larger perceived gap. In such a situation, response precision could certainly suffer. In Agaoglu et al., while the target was very similar to ours (although their oriented bar was almost twice as long as ours), they did not use a four dot mask. Rather, they compared masking functions between an annulus mask, a noise mask, and a structure mask (the last of which comprised three bars similar to the target bar but in random orientations). It is easy to conceive how, for example, their structure mask could add noise in the orientation encoding domain. Furthermore, the parameter in these masking functions was the SOA of a pulsed mask, rather than the mask duration of a common onset mask. Finally, in both these studies, stimuli were presented non foveally. In Harrison et al., the target was one among anywhere from two to eight possible targets presented at 3.5 degrees from fixation. In Agaoglu et al., a single target was presented at a 6 degree horizontal eccentricity. While orientation discrimination thresholds do rise as a function of eccentricity (Sally \& Gurnsey, 2004), this alone does not explain why precision would be reduced with the addition of a para or metacontrast mask, as shown in Agaoglu et al., or with an increased four dot mask duration, as in Harrison et al. However, there may be an interaction between contrast and eccentricity. In Mareschal \& Shapley (2004), the effect of contrast upon orientation discrimination thresholds was evident at larger target sizes in the periphery compared to the fovea. When stimuli were presented at 5 degrees of horizontal eccentricity, there was a large effect of contrast upon orientation discrimination of gratings that were as large as 1 degree, whereas in the fovea, all observers showed contrast invariance at this stimulus size. In Agaoglu et al., the oriented bars were 1 degree in length, and were presented at 6 degrees of eccentricity. If their masks were operating through contrast reduction, then this is a plausible explanation of the reduced response precision.

The current study reinforces Filmer et al.'s (2015) finding that four dot masking can be reliably obtained with a fully attended and centrally presented target, an idea which must be accounted for in any theory of masking via object updating. The direct comparison between the two mask types used in our study adds new evidence that four dot masking operates via unique mechanisms when compared to traditional masking stimuli. Our last experiment provides novel insights into the way in which four dot masking impacts the visual representation of a foveated target.

On a final note, we have chosen to refrain from making assumptions about the underlying mechanisms of masking, beyond those that can be reasonably supported by our data, and this is reflected in our terminology throughout this article. While our findings are consistent with an object mediated account of masking, we have not introduced any experimental manipulations that test, for example, whether object updating (versus object substitution) accounts for our masking (Enns, Lleras, $\&$ Moore, 2010). Accordingly, we have avoided the use of the terms object substitution and object updating wherever possible. Similarly, as our data say nothing about the relationship between feedforward and feedback signals (Kafaligonul, Breitmeyer, \& Öğmen, 2015), we have avoided implicitly assuming any particular processing regime. 


\section{Appendix}

378

379

380

381

382

383

384

385

386
Our study used a relatively small number of observers for each experiment. In addition to this, our finding in Experiment 3, which showed no evidence for a change in response precision with a 250 ms mask, does not allow us to meaningfully quantify the evidence that there was no change in precision. To address this, we conducted a Bayesian analysis of our data, using the free sofware JASP, version 0.7.5.5 (Love et al., 2015; Morey \& Rouder, 2015). This software calculates the Bayes factors on distributions of effect size to assess the relative probability of observed data, between two competing statistical hypotheses (for an introduction to Bayes factors, see Jarosz \& Wiley, 2014). We have included the JASP analysis files with this submission.

\section{Experiment 1}

To assess the evidence for and against a masking effect at a mask duration of $250 \mathrm{~ms}$, we conducted a Bayesian paired samples t-test (Rouder, Speckman, Sun, Morey, \& Iverson, 2009) between the common offset and $250 \mathrm{~ms}$ conditions, with the default Cauchy prior width of 0.707 (centered on an effect size of 0). The results, shown below in Figure A1, show a moderate preference for the alternative hypothesis over the null hypothesis by a Bayes factor of 8.62. This supports the idea that the four dot mask had an impact upon performance. 


\begin{tabular}{|c|c|c|}
\hline & $\mathrm{BF}_{10}$ & error $(\%)$ \\
\hline Alternative Hypothesis: Common Offset $\neq 250 \mathrm{~ms}$ & 8.62 & $1.12 \times 10^{-9}$ \\
\hline
\end{tabular}
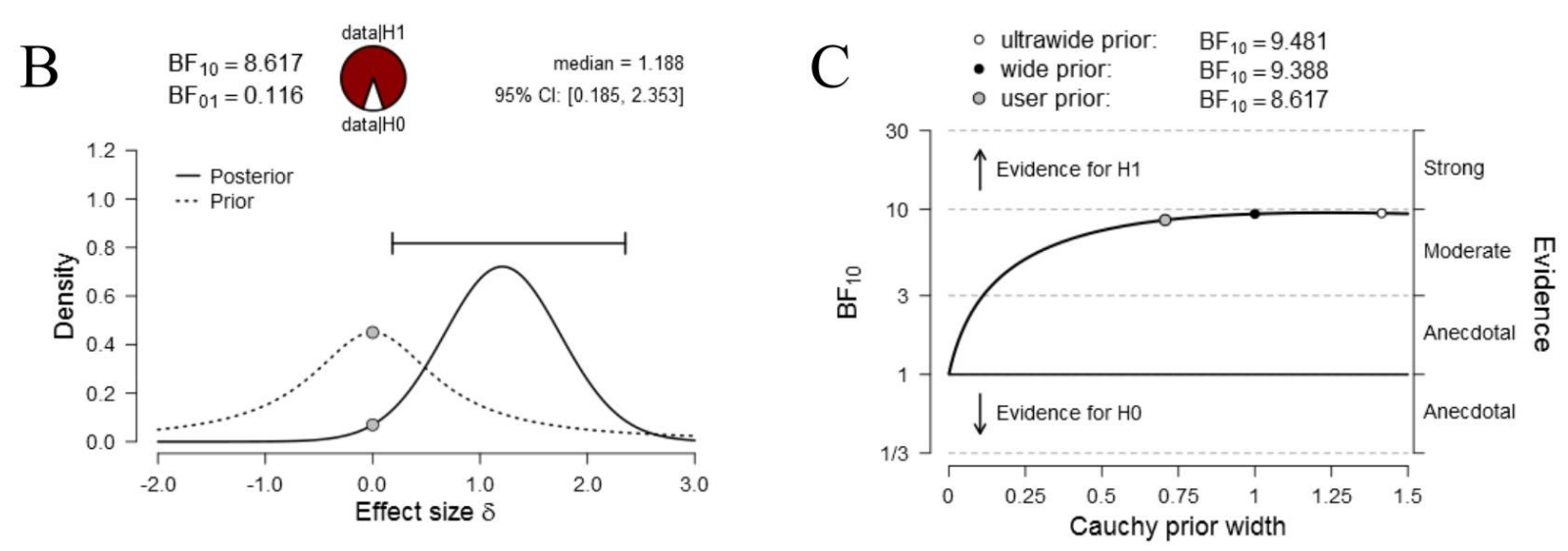

Figure A1. A Bayesian paired samples t-test comparing baseline to the $250 \mathrm{~ms}$ condition in Experiment 1. A: $\mathrm{BF}_{10}$ denotes the Bayes factor for the alternative hypothesis over the null. Here, the alternative hypothesis is that performance in baseline is different from that in the $250 \mathrm{~ms}$ condition. The null is that performance is equal in these two conditions. The error column indicates the error in estimating the Bayes factor, as a percentage. Shown in $\mathbf{B}$ are both the posterior and prior distributions of effect size. The null hypothesis defines an effect size of 0 , depicted here in filled grey circles. $\mathbf{C}$ shows the robustness of the Bayes factor as a function of the prior width. The small grey circle depicts the Bayes factor at the default prior with of 0.707 . The curve here shows that the Bayes factor remains relatively stable until much lower widths are used. $\mathbf{B}$ and $\mathbf{C}$ were generated using the JASP software.

\section{Experiment 2}

We ran a Bayesian repeated measures ANOVA (Rouder, Morey, Speckman, \& Province, 2012), with mask type as the first factor: two levels (4DM, annulus); second factor = target-mask separation: four levels, excluding baseline), using the default set of priors (which assumes all five models are equally probable). The results are shown below in Figure A2. While the interaction model outperformed all the other models, relative to the null, it only outperformed the Mask Type + Separation model by a factor of 1.23 , which is weak evidence in favour of this interaction. While this analysis is qualitatively consistent with our earlier analysis, it does not provide strong support for the 
401 interaction.

\begin{tabular}{lcccc} 
Bayesian repeated measures ANOVA & \\
\hline Models (all models include subject as a factor) & $\mathrm{P}(\mathrm{M})$ & $\mathrm{P}(\mathrm{M} \mid \mathrm{data})$ & $\mathrm{BF}_{10}$ & error $(\%)$ \\
\hline Null model & 0.2 & 0.118 & 1.000 & \\
Mask Type & 0.2 & 0.120 & 1.021 & 0.831 \\
Separation & 0.2 & 0.205 & 1.743 & 0.551 \\
Mask Type + Separation & 0.2 & 0.250 & 2.118 & 1.125 \\
Mask Type + Separation + Mask Type x Separation & 0.2 & 0.307 & 2.606 & 2.272 \\
\end{tabular}

Figure A2. Results from the Bayesian repeated measures ANOVA for data from Experiment 2. The first column indicates the model being tested. $\mathrm{P}(\mathrm{M})$ indicate prior odds; $\mathrm{P}(\mathrm{M} \mid$ data $)$ indicate posterior odds, and $\mathrm{BF}_{10}$ indicates the Bayes factor of the model relative to the null. Due to the transitivity of Bayes factors, models can be directly compared by taking a ratio of their Bayes factors. For example, the interaction model $\left(\mathrm{BF}_{10}=2.606\right)$ outperforms the two factor model $\left(\mathrm{BF}_{10}=2.118\right)$ by a factor of $2.606 / 2.118=1.23$.

\section{Experiment 3}

We provide here three separate analyses. First, we compared the overall errors in the common offset vs. $250 \mathrm{~ms}$ conditions, using a Bayesian paired samples t-test. Second, we ran a Bayesian repeated measures ANOVA to test for the interaction between target orientation (oblique, cardinal) and mask condition (common offset, $250 \mathrm{~ms}$ ). Finally, we compared the parameters of our mixture model (weight of Gaussian, and Sigma) between the common offset and $250 \mathrm{~ms}$ conditions using Bayesian paired samples t-tests. The same prior defaults were used as in the Bayesian analyses for the first two experiments. The results, shown below in Figure A3, are consistent with our earlier analyses. There was strong evidence for masking, with a Bayes factor of 15.36 favouring the alternative hypothesis (overall errors in common offset and $250 \mathrm{~ms}$ conditions are different) over the null. In the repeated measures ANOVA, the target orientation + mask condition model was favoured over the interaction 
413 model by a factor of 2.41, which is weak evidence against an interaction between target orientation and 414 mask condition. This lends support, albeit weak, to the idea that masking here is independent of the 415 relationship between target orientation and dot location. Finally, the paired samples t-tests showed very 416 strong evidence that the weight of the Gaussian component was different between the two masking 417 conditions (Bayes factor $=27.75$ favouring alternative over null), and moderate evidence that the 418 standard deviation of this Gaussian component did not change (Bayes factor $=3.07$, favouring the null 419 over the alternative). This is consistent with our earlier interpretation, and provides direct evidence that the precision of observer responses did not change between masking conditions.

\begin{tabular}{|c|c|c|c|c|}
\hline Bayesian paired samples t-tests & $\mathrm{BF}_{10}$ & error $(\%)$ & & \\
\hline Alternative Hypothesis: Com Offset Err $\neq 250 \mathrm{~ms}$ Err & 15.36 & $3.60 \times 10^{-6}$ & & \\
\hline Alternative Hypothesis: Com Offset $\mathrm{W}_{\mathrm{G}} \neq 250 \mathrm{~ms} \mathrm{~W}_{\mathrm{G}}$ & 27.75 & $2.146 \times 10^{-6}$ & & \\
\hline Alternative Hypothesis: Com Offset $\sigma \neq 250 \mathrm{~ms} \sigma$ & 0.326 & $5.51 \times 10^{-5}$ & & \\
\hline \multicolumn{5}{|l|}{ Bayesian repeated measures ANOVA } \\
\hline Models (all models include subject as a factor) & $\mathrm{P}(\mathrm{M})$ & $\mathrm{P}(\mathrm{M} \mid$ data $)$ & $\mathrm{BF}_{10}$ & error $(\%)$ \\
\hline Null model & 0.2 & 0.064 & 1.000 & \\
\hline Mask Condition & 0.2 & 0.055 & 0.863 & 0.957 \\
\hline Orientation & 0.2 & 0.345 & 5.382 & 1.506 \\
\hline Mask Condition + Orientation & 0.2 & 0.379 & 5.910 & 4.664 \\
\hline Mask Condition + Orientation + Mask Condition $x$ Orientation & 0.2 & 0.157 & 2.449 & 5.001 \\
\hline
\end{tabular}

Figure A3. A: Three Bayeseian paired samples t-tests are shown here. The first is the overall errors between the common offset baseline and the $250 \mathrm{~ms}$ mask conditions. Next is the weights of the Gaussian components of the mixture model analysis between these two conditions. The third is the standard deviation of these Gaussian components. Note the Bayes factor here is with respect to the alternative hypothesis relative to the null hypothesis. The reciprocal of this value $\left(\mathrm{BF}_{01}\right)$ represents the likelihood of our data given the null hypothesis relative the likelihood of our data given the alternative hypothesis. B: Results of the Bayesian repeated measures ANOVA on the effects of target orientation and masking condition. Note that the interaction model has a smaller Bayes factor than that of the two factor model.

\section{Acknowledgements}


We would like to thank the editor and the two reviewers for their time and effort. Their critical insights greatly helped improve upon earlier versions of the manuscript.

\section{References}

Agaoglu, S., Agaoglu, M. N., Breitmeyer, B., \& Öğmen, H. (2015). A statistical perspective to visual masking. Vision Research, 115(Pt A), 23-39. http://doi.org/10.1016/j.visres.2015.07.003

Argyropoulos, I., Gellatly, A., Pilling, M., \& Carter, W. (2013). Set size and mask duration do not interact in object-substitution masking. Journal of Experimental Psychology. Human Perception and Performance, 39(3), 646-61. http://doi.org/10.1037/a0030240

Breitmeyer, B., \& Öğmen, H. (2006). Visual Masking: Time slices through conscious and unconscious vision. Applied Cognitive Psychology (Vol. 21). Oxford University Press. http://doi.org/10.1093/acprof:oso/9780198530671.001.0001

Camp, S. J., Pilling, M., Argyropoulos, I., \& Gellatly, A. (2015). The role of distractors in object substitution masking. Journal of Experimental Psychology. Human Perception and Performance, 41(4), 940-57. http://doi.org/10.1037/xhp0000065

Choo, H., \& Franconeri, S. L. (2010). Objects with reduced visibility still contribute to size averaging. Attention, Perception \& Psychophysics, 72(1), 86-99. http://doi.org/10.3758/APP.72.1.86

Di Lollo, V., Enns, J. T., \& Rensink, R. A. (2000). Competition for consciousness among visual events: the psychophysics of reentrant visual processes. Journal of Experimental Psychology. General, 129(4), 481-507. http://doi.org/10.1016/j.visres.2003.10.024

Dux, P. E., Visser, T. A., Goodhew, S. C., \& Lipp, O. V. (2010). Delayed reentrant processing impairs visual awareness an object-substitution-masking study. Psychological Science, 21(9), 1242-1247. http://doi.org/10.1177/0956797610379866

Elze, T. (2010). Misspecifications of Stimulus Presentation Durations in Experimental Psychology: A Systematic Review of the Psychophysics Literature. PLoS ONE, 5(9), e12792. http://doi.org/10.1371/journal.pone.0012792

Enns, J. T. (2004). Object substitution and its relation to other forms of visual masking. Vision Research, 44(12), 1321-31. http://doi.org/10.1016/j.visres.2003.10.024

Enns, J.T., Lleras, A., \& Moore, C.M. (2010). Object updating: A force for perceptual continuity and scene stability in human vision. In R. Nijhawan, \& B. Khurana (Ed.), Space and time in perception and action (pp. 503-520). Cambridge, MA: Cambridge University Press.

Filmer, H. L., Mattingley, J. B., \& Dux, P. E. (2015). Object substitution masking for an attended and 
454

455

456

457

458

459

460

461

462

463

464

465

466

467

468

469

470

471

472

473

474

475

476

477

478

479

480

481

482

483

484

485

486

487

foveated target. Journal of Experimental Psychology. Human Perception and Performance, 41(1), 6-10. http://doi.org/10.1037/xhp0000024

Goodhew, S. C., Dux, P. E., Lipp, O. V, \& Visser, T. a W. (2012). Understanding recovery from object substitution masking. Cognition, 122(3), 405-15. http://doi.org/10.1016/j.cognition.2011.11.010

Goodhew, S. C., Edwards, M., Boal, H. L., \& Bell, J. (2015). Two Objects or One? Similarity Rather Than Complexity Determines Objecthood When Resolving Dynamic Input. Journal of Experimental Psychology. Human Perception \& Performance, 41(1), 102-10. http://doi.org/10.1037/xhp0000022

Goodhew, S. C., Pratt, J., Dux, P. E., \& Ferber, S. (2013). Substituting objects from consciousness: a review of object substitution masking. Psychonomic Bulletin \& Review, 20(5), 859-77. http://doi.org/10.3758/s13423-013-0400-9

Goodhew, S. C., Visser, T. a W., Lipp, O. V, \& Dux, P. E. (2011). Implicit semantic perception in object substitution masking. Cognition, 118(1), 130-4. http://doi.org/10.1016/j.cognition.2010.10.013

Harrison, G. W., Rajsic, J., \& Wilson, D. E. (2016). Object-substitution masking degrades the quality of conscious object representations. Psychonomic Bulletin \& Review, 2016, 23(1):180-186. http://doi.org/10.3758/s13423-015-0875-7

Jarosz, A. F., \& Wiley, J. (2014). What are the odds? A practical guide to computing and reporting Bayes Factors. The Journal of Problem Solving, 7(1), 2-9. http://dx.doi.org/10.7771/19326246.1167

Jiang, Y., \& Chun, M. M. (2001). The spatial gradient of visual masking by object substitution. Vision Research, 41(24), 3121-31. http://doi.org/10.1016/S0042-6989(01)00218-8

Kafaligonul, H., Breitmeyer, B. G., \& Öğmen, H. (2015). Feedforward and feedback processes in vision. Frontiers in Psychology, 6, 279. http://doi.org/10.3389/fpsyg.2015.00279

Kahan, T. A., \& Enns, J. T. (2010). Object trimming: When masking dots alter rather than replace target representations. Journal of Experimental Psychology. Human Perception and Performance, 36(1), 88-102. http://doi.org/10.1037/a0016466

Lleras, A., \& Moore, C. M. (2003). When the target becomes the mask: using apparent motion to isolate the object-level component of object substitution masking. Journal of Experimental Psychology. Human Perception and Performance, 29(1), 106-20. http://doi.org/10.1037/00961523.29.1.106

Love, J., Selker, R., Marsman, M., Jamil, T., Dropmann, D., Verhagen, A. J., Ly, A., Gronau, Q. F., Smira, M., Epskamp, S., Matzke, D., Wild, A., Knight, P., Rouder, J. N., Morey, R. D., \& Wagenmakers, E.-J. (2015). JASP (Version 0.7.5) [Computer software]

Macknik, S. L., \& Livingstone, M. S. (1998). Neuronal correlates of visibility and invisibility in the 
primate visual system. Nature Neuroscience, 1(2), 144-9. http://doi.org/10.1038/393

Mareschal, I., \& Shapley, R. M. (2004). Effects of contrast and size on orientation discrimination. Vision Research, 44(1), 57-67. http://doi.org/10.1016/j.visres.2003.07.009

491 Morey, R. D., \& Rouder, J. N. (2015). BayesFactor (Version 0.9.11-3) [Computer software].

492 Myung, I. J. (2003). Tutorial on maximum likelihood estimation. Journal of Mathematical Psychology, 493 47(1), 90-100. http://doi.org/10.1016/S0022-2496(02)00028-7

494

Pilling, M., \& Gellatly, A. (2010). Object substitution masking and the object updating hypothesis. Psychonomic Bulletin \& Review, 17(5), 737-42. http://doi.org/10.3758/PBR.17.5.737

Pilling, M., Gellatly, A., Argyropoulos, Y., \& Skarratt, P. (2014). Exogenous spatial precuing reliably modulates object processing but not object substitution masking. Attention, Perception, \& Psychophysics, 76, 1560-76. http://doi.org/10.3758/s13414-014-0661-z

Rouder, J. N., Speckman, P. L., Sun, D., Morey, R. D., \& Iverson, G. (2009). Bayesian t tests for accepting and rejecting the null hypothesis. Psychonomic Bulletin \& Review, 16(2), 225-237.

Rouder, J. N., Morey, R. D., Speckman, P. L., \& Province, J. M. (2012). Default Bayes factors for ANOVA designs. Journal of Mathematical Psychology, 56(5), 356-374.

Sally, S. L., \& Gurnsey, R. (2004). Orientation discrimination across the visual field: matching perceived contrast near threshold. Vision Research, 44(23), 2719-27. http://doi.org/10.1016/j.visres.2004.06.004

Shooner, C., Tripathy, S. P., Bedell, H. E., \& Öğmen, H. (2010). High-capacity, transient retention of direction-of-motion information for multiple moving objects. Journal of Vision, 10(6):8, 1-20.

Tata, M. S., \& Giaschi, D. E. (2004). Warning: attending to a mask may be hazardous to your perception. Psychonomic Bulletin \& Review, 11(2), 262-8. http://doi.org/10.3758/BF03196568

Taylor, M., \& Creelman, C. D. (1967). PEST: Efficient estimates on probability functions. The Journal of the Acoustical Society of America, 41(4A), 782-787. http://doi.org/10.1121/1.1910407

Zhang, W., \& Luck, S. J. (2008). Discrete fixed-resolution representations in visual working memory. Nature, 453(7192), 233-235. doi:10.1038/nature06860 